\title{
COHOMOLOGICAL LOCAL CONNECTEDNESS OF DECOMPOSITION SPACES
}

\author{
JERZY DYDAK AND JOHN J. WALSH
}

(Communicated by James E. West)

\begin{abstract}
For a map $f: X \rightarrow Y$, let $\mathscr{H}^{k}[f]$ denote the associated $k$-dimensional cohomology sheaf. The main result is that, for a proper map between locally compact metrizable spaces, if the sheaves $\mathscr{H}^{k}[f]$ are locally constant and $X$ is cohomologically locally connected, then $Y$ is cohomologically locally connected. The result can be viewed as a variation on a number of similar results dating to work of Vietoris. The setting for this paper is quite general and the proof is not difficult, involving a routine analysis using the Leray-Grothendieck spectral sequence. Versions of known comparable results for homotopical local connectedness can be recovered by combining the result with standard universal coefficient theorems that translate cohomological information to homological information and with a local Hurewicz theorem.
\end{abstract}

\section{INTRODUCTION}

There is an extensive literature that, in the setting of a proper map $f: X \rightarrow$ $Y$, provides conditions on the mapping $f$ to ensure that the local connectivity properties of $X$ are inherited by $Y$. An early occurrence, a consequence of the Vietoris-Begle Mapping Theorem [Be], is that if $f$ is acyclic and $X$ is homologically locally connected, then $Y$ is homologically locally connected. A similar result, dealing with homotopical local connectedness, was established by Smale $[\mathrm{Sm}]$ and extended by various authors, including [Dug], [Koz], [Dy $\left.{ }_{2}\right]$, [CD], [AP], and [Ar]. With the exception of [CD], the hypotheses centered on the triviality of the homology/cohomology groups or homotopy groups (perhaps measured with respect to a system of neighborhoods) of the point-inverses $f^{-1}(y)$, for $y \in Y$.

More recently, the first author and J. Segal [DS], advancing a point of view found in $[C D]$, replaced the assumption that the groups of each point-inverse be trivial, with the much less restrictive assumption that these groups align in a coherent manner. The approach used in the latter work involves mingling

Received by the editors September 26, 1988.

1980 Mathematics Subject Classification (1985 Revision). Primary 55N30, 54C10, 55T99.

Key words and phrases. Cohomological local connectedness, proper mapping, LerayGrothendieck spectral sequence..

Both authors supported in part by grants from the National Science Foundation. 
of hypotheses involving the homology groups of the point-inverses with the assumption that the point-inverses are nearly 1-movable, the latter a condition that ensures that the image is homotopically locally 1 -connected $\left[\mathrm{Dy}_{1}\right]$. The conclusions reached in [DS] involve the homotopical local connectivity of the image. An alternate approach, presented herein, entails an analysis that involves only homological information, both in the hypotheses and conclusion (in fact, cohomology rather than homology seems most natural). In standard fashion, results on homotopical local connectedness can be recovered using a local version of the Hurewicz theorem [Hu]. For example, as recorded in [DaW], the local Hurewicz theorem establishes that the combination of the Vietoris-Begle Mapping Theorem and the work of Dydak implies that acyclic maps having nearly 1-movable point-inverses preserve homotopical local connectedness.

This paper emerged from an analysis of the first author's work with J. Segal in [DS], where the setting is mappings $f: X \rightarrow Y$ between locally compact spaces and where the conditions are, first, that the point-inverses of $f$ be $L C^{n}$ divisors (roughly, that the point-inverses have finitely generated homology and are nearly 1-movable), and, second, that the map $f$ is homology $k$-stable (intuitively, that the homology groups of the point-inverses are aligned in a locally constant manner). The conclusions in [DS] are that $Y$ is homotopically locally connected (in dimensions that depend on the choice of $n$ and $k$ ). One drawback of mixing homological and homotopical information is that the proof in [DS] is very involved. The approach used in this paper is to "dualize" and use cohomology in place of homology, thereby obtaining a purely cohomological result. The concept of homology $k$-stable dualizes to a standard notion that the associated cohomology sheaf $\mathscr{H}^{f}[f]$ is locally constant. Furthermore, the proof reduces to a routine use of the Leray-Grothendieck spectral sequence.

\section{Terminology AND the LeRAy-Grothendieck SPECTRAL SEQUENCE}

For the sake of simplicity of presentation, the coefficient ring for cohomology is the integers $\mathbf{Z}$ and is normally suppressed. The cohomology theory used is Cech theory and the cohomology of a space $X$ with integer coefficients is denoted $H^{k}(X)$. For a sheaf $\mathscr{F}$ of abelian groups on a space $X$, the cohomology of $X$ with coefficients in $\mathscr{F}$ is denoted $H^{k}(X ; \mathscr{F})$. The group $H^{0}(X ; \mathscr{F})$ can be identified with the group of sections $\Gamma(X, \mathscr{F})$. Spaces are assumed to be metrizable.

A space $X$ is cohomologically $n$-connected at $x \in X$, denoted $n$-clc at $x \in$ $X$, provided, for each neighborhood $U$ of $x$, there is a neighborhood $V \subset U$ of $x$ such that the inclusion induced homomorphism $\widetilde{H}^{n}(U) \rightarrow \widetilde{H}^{n}(V)$ is the zero homomorphism (where $\widetilde{H}^{*}$ denotes reduced cohomology). A space $X$ is $c l c^{n}$ provided $X$ is $k$-clc at each point $x \in X$ for $k \leq n$.

For a map $f: X \rightarrow Y$ and integer $k \geq 0$, there is a presheaf on $Y$ that assigns to an open subset $U \subset Y$ the cohomology group $H^{k}\left(f^{-1}(U)\right)$ and assigns 
to an inclusion $V \hookrightarrow U$ the inclusion-induced homomorphism $H^{k}\left(f^{-1}(U)\right) \rightarrow$ $H^{k}\left(f^{-1}(V)\right)$. The associated sheaf is denoted $\mathscr{H}^{k}[f]$ and, for a proper map $f$, i.e., $f^{-1}(K)$ is compact for each compact subset $K$, the stalk of $\mathscr{H}^{k}[f]$ at a point $y \in Y$ is the cohomology group $H^{k}\left(f^{-1}(y)\right)$.

The next result and the comments following it record information related to the Leray-Grothendieck spectral sequence, specifically, information needed in this paper. For additional details, the reader is referred to [Gr], [Sw], and $[\mathrm{Br}]$.

Theorem (Leray-Grothendieck). For a map $f: X \rightarrow Y$, there is a first quadrant spectral sequence $\left\{E_{r}=E_{r}(f): r \geq 2\right\}$ such that:

(1) $E_{2}^{p, q}=H^{p}\left(Y ; \mathscr{H}^{q}[f]\right)$,

(2) $E_{\infty}^{p, q}$ is associated to a filtration of $H^{p+q}(X)$,

(3) the edge homomorphism is $f^{*}: H^{p}\left(Y ; f_{*} Z\right) \cong E_{2}^{p, 0} \Rightarrow E_{\infty}^{p, 0} \hookrightarrow H^{p}(X)$.

Remarks. (a) Formally, $f_{*} \mathbf{Z}$ is the sheaf determined by the presheaf that assigns to an open subset $U \subset Y$ the sections over $f^{-1}(U)$ of the constant sheaf $X \times \mathbf{Z}$. The sheaf $f_{*} \mathbf{Z}$ is canonically isomorphic to the sheaf $\mathscr{H}^{0}[f]$. In the situations that arise in this paper, the sheaf $\mathscr{H}^{0}[f]$ is locally constant. For example, if $Y$ is locally connected, $f$ is proper, and $f^{-1}(y)$ is connected for each $y \in Y$, then $\mathscr{H}^{0}[f]$ is the constant sheaf $Y \times \mathbf{Z}$ on $Y$; and hence, the cohomology group $H^{p}\left(Y ; f_{*} \mathbf{Z}\right)$ is the usual cohomology of $Y$.

(b) $E_{r}^{p, q}=\operatorname{ker}\left(d_{r}\right) / \operatorname{im}\left(d_{r}\right)$ where the differential $d_{r}^{p, q}: E_{r}^{p, q} \rightarrow E_{r}^{p+r, q-r+1}$ has bidegree $(r, 1-r)$.

(c) $E_{r}^{p, q}=E_{r+1}^{p, q}=\cdots=E_{\infty}^{p, q}$ for $p \geq 1$ and $r \geq p+q+1$ (when $p=0$, $E_{r}^{0, q}=E_{r+1}^{0, q}=\cdots=E_{\infty}^{0, q}$ for $\left.r \geq q+2\right)$.

(d) The filtration is

$$
0 \subset J_{0} \subset J_{1} \subset \cdots \subset J_{p}=H^{p}(X)
$$

where $J_{0}=E_{\infty}^{p, 0}$ and $J_{i} / J_{i-1}=E_{\infty}^{p-i, i}$ for $i \leq p$.

\section{THE PRINCIPAL RESULT}

Theorem 1 below forms the core of the paper, and its proof illustrates a use of the computational machinery that the Leray-Grothendieck spectral sequence makes available. At first glance, it might appear that the hypotheses of the theorem do not include that the point-inverses of $f$ have finitely generated cohomology. However, the local constancy of the cohomology sheaves implies that at points $y \in Y$ satisfying $(*)$, the cohomology of $f^{-1}(y)$ is finitely generated (in dimensions $<n$ ). Of course, in the application stated in Corollary 1 as well as those presented in $\S 3$, an assumption of finite generation is either explicitly or implicitly present. 
There is one consequence of the assumption of cohomologically locally connected that is needed. An elementary proof, using only the Mayer-Vietoris sequence, can be found in [Br, pp. 78-80].

Lemma 0. If $X$ is a locally compact space that is $\mathrm{clc}^{n}$ and $K \subset U$, where $K$ is compact and $U$ is open, then the image of $H^{k}(U) \rightarrow H^{k}(K)$ is finitely generated for $k \leq n$.

The following lemma contains a purely algebraic result that is used in the proof of Theorem 1.

Lemma 1. If $f: G \rightarrow H$ and $g: H \rightarrow F$ are homomorphisms of abelian groups and there are subgroups $G_{1} \subset G$ and $H_{1} \subset H$ such that $f\left(G_{1}\right) \subset H_{1}, g\left(H_{1}\right)$ is finitely generated, and the image of $G / G_{1} \rightarrow H / H_{1}$ is finitely generated, then $g f(G)$ is finitely generated.

Proof. Choose elements $h_{1}, h_{2}, \ldots, h_{s} \in H$ so that their images in $H / H_{1}$ generate the image of $G / G_{1}$, and choose elements $f_{1}, f_{2}, \ldots, f_{t} \in F$ that generate $g\left(H_{1}\right)$. Let $F_{1}$ be the subgroup of $F$ generated by the $f_{i}$ 's and the $g\left(h_{i}\right)$ 's. The factoring

$$
G \rightarrow G / G_{1} \rightarrow H / H_{1} \rightarrow F / F_{1}
$$

detects that the image of $G$ in $F / F_{1}$ is trivial and, therefore, $g f(G)$ is contained in the finitely generated subgroup $F_{1}$.

Theorem 1. If $f: X \rightarrow Y$ is a proper map between locally compact metrizable spaces, $X$ is clc ${ }^{n+1}$, and the cohomology sheaves $\mathscr{H}^{0}[f]$ as well as $\mathscr{H}^{k}[f]$, for $k \leq n-1$, are locally constant, then $Y$ is $(n+1)$-clc at each point $y$ satisfying:

(*) for each $k \leq n$ and each neighborhood $U$ of $y$, there is a neighborhood $V$ of $y$ such that the image of $\Gamma_{k}(U) \rightarrow \Gamma_{k}(V)$ is finitely generated (where $\Gamma_{k}(W)$ is the group of sections of $\mathscr{H}^{k}[f]$ over the open subset $\left.W \subset Y\right)$.

Proof. Proceeding by induction on $n$, starting with $n=-1$, which is the wellknown result that the $Y$ is locally connected since $X$ is locally connected, assume that $Y$ is $\operatorname{clc}^{n}$ at each point satisfying condition (*). Assume that $y_{0} \in Y$ satisfies condition $(*)$, and replacing $Y$ by a neighborhood of $y_{0}$ if necessary, assume that each of the sheaves $\mathscr{H}^{0}[f]$ and $\mathscr{H}^{k}[f]$, for $k \leq n-1$, is constant. The constancy of these sheaves means, for a choice of $V \subset U$ as in $(*)$, that $H^{k}\left(f^{-1}\left(y_{0}\right)\right)$ is isomorphic to a subgroup of the image of $\Gamma_{k}(U) \rightarrow$ $\Gamma_{k}(V)$ for $k \leq n-1$. Thus $H^{k}\left(f^{-1}\left(y_{0}\right)\right)$ is finitely generated for $k<n$, as was observed in the discussion at the beginning of the section. (Since $\mathscr{H}^{0}[f]$ is assumed locally constant, in case $n=0, H^{0}\left(f^{-1}\left(y_{0}\right)\right)$ is finitely generated as well.)

Since $Y$ is clc ${ }^{n}$ at $y_{0}$, the universal coefficient theorem for Čech cohomology [Sp] establishes that $Y$ is $\mathrm{clc}^{n-1}$ at $y_{0}$ with respect to any coefficient group. That is, for any neighborhood $U$ of $y_{0}$, there is a neighborhood $V \subset U$ of 
$y_{0}$ such that the inclusion-induced homomorphism $H^{k}(U ; G) \rightarrow H^{k}(V ; G)$ is trivial for each abelian group $G$ and for $k \leq n-1$.

For a subset $A \subset Y$, the terms in the Leray-Grothendieck spectral sequence for the restriction $f^{-1}(A) \rightarrow A$ will be denoted ${ }_{A} E_{r}^{p, q}$ and the differentials denoted ${ }_{A} d_{r}^{p, q}$.

For each open neighborhood $U$ of $y_{0}$, specify a neighborhood $\alpha(U)$ of $y_{0}$ with $\mathrm{cl}(\alpha(U)) \subset U$ satisfying:

(1) the image of $\Gamma_{n}(U) \rightarrow \Gamma_{n}(\alpha(U))$ is finitely generated;

(2) the inclusion-induced homomorphism $H^{k}(U ; G) \rightarrow H^{k}(\alpha(U) ; G)$ is trivial for $k \leq n-1$ and for all abelian groups $G$;

(3) the image of $H^{n+1}\left(f^{-1}(U)\right) \rightarrow H^{n+1}\left(f^{-1}(\alpha(U))\right)$ is finitely generated.

As is recorded in Lemma 0 , the last condition is a consequence of the assumption that $X$ is $\mathrm{clc}^{n+1}$. Since $\mathscr{H}^{k}[f]$ is constant for $k \leq n-1$, $H^{p}\left(A ; \mathscr{H}^{k}\left[\left.f\right|_{A}\right]\right)=H^{p}\left(A ; H^{k}\left(f^{-1}\left(y_{0}\right)\right), \quad\right.$ for $k \leq n-1$ and for subsets $A \subset Y$.

Therefore, in the presence of condition 2, for each $V \subset \alpha(U)$, the homomorphism

$$
{ }_{U} E_{2}^{p, q} \rightarrow{ }_{V} E_{2}^{p, q}, \quad \text { and , hence, }{ }_{U} E_{r}^{p, q} \rightarrow_{V} E_{r}^{p, q}, \quad \text { for } r \geq 2,
$$

is trivial for $p, q \leq n-1$.

A consequence of condition 1 and the identification

$$
{ }_{U} E_{2}^{0, n}=H^{0}\left(U ; \mathscr{C}^{n}\left[\left.f\right|_{f^{-1}(U)}\right]\right)=\Gamma_{n}(U)
$$

is that the image of

$$
{ }_{U} E_{2}^{0, n} \rightarrow{ }_{V} E_{2}^{0, n}, \quad \text { and hence of }{ }_{U} E_{r}^{0, n} \rightarrow{ }_{V} E_{r}^{0, n}, \quad \text { for } r \geq 2,
$$

is finitely generated.

A consequence of condition 3 and the filtration associated to the spectral sequence (see the remarks in $\S 1$ ) is that the image of the inclusion induced homomorphism

$$
{ }_{U} E_{\infty}^{p, q} \rightarrow{ }_{V} E_{\infty}^{p, q}
$$

is finitely generated for $p+q=n+1$. Since $E_{\infty}^{p, q}=E_{p+q+1}^{p, q}$, for $p \geq 1$ (see Remark (c) in $\S 1)$, the image of

$$
{ }_{U} E_{n+2}^{p, q} \rightarrow{ }_{V} E_{n+2}^{p, q}
$$

is finitely generated for $p \geq 1$ and $p+q=n+1$ as well.

The remainder of the proof is to show, by induction, that for each neighborhood $U$ of $y_{0}$ and each integer $0 \leq m \leq n$, there is a neighborhood $V \subset U$ of $y_{0}$ such that the image of

$$
{ }_{U} E_{n+2-m}^{n+1,0} \rightarrow{ }_{V} E_{n+2-m}^{n+1,0}
$$


is finitely generated. For $m=0, V=\alpha(U)$ was just shown to have this property. Inductively, suppose that $1 \leq m \leq n$ and that $W \subset U$ are open neighborhoods of $y_{0}$ such that $W \subset \alpha(U)$ and the image of

$$
{ }_{U} E_{n+2-m}^{n+1,0} \rightarrow{ }_{W} E_{n+2-m}^{n+1,0}
$$

is finitely generated. Specify a neighborhood $V \subset \alpha(W)$ and set

$$
\begin{gathered}
G={ }_{U} E_{n+2-(m+1)}^{n+1,0}, \quad H={ }_{W} E_{n+2-(m+1)}^{n+1,0}, \quad F={ }_{V} E_{n+2-(m+1)}^{n+1,0}, \\
G_{1}=\operatorname{im}\left({ }_{U} d_{n+2-(m+1)}^{m, n-m}\right), \quad H_{1}=\operatorname{im}\left({ }_{W} d_{n+2-(m+1)}^{m, n-m}\right),
\end{gathered}
$$

and let $f: G \rightarrow H$ and $g: H \rightarrow F$ be the inclusion-induced homomorphisms. Naturality ensures that $f\left(G_{1}\right) \subset H_{1}$ and ( $\left.\ddagger\right)$ records that the image of $G / G_{1} \rightarrow$ $H / H_{1}$ is finitely generated. The finite generation of $g\left(H_{1}\right)$ is a consequence of the commuting diagram

$$
\begin{aligned}
& { }_{W} E_{n+2-(m+1)}^{n+1,0} \longrightarrow{ }_{V} E^{E_{n+2-(m+1)}^{n+1,0}} \\
& \uparrow u \cdot d_{n+2-(m+1)}^{m, n-m} \quad \uparrow \cdot d_{n+2-(m+1)}^{m, n-m} \\
& { }_{W} E_{n+2-(m+1)}^{m, n-m} \longrightarrow{ }_{V} E_{n+2-(m+1)}^{m, n-m}
\end{aligned}
$$

and of the information recorded in (b) for $m=0$ and in (a) for $n-1 \geq m \geq 1$. In turn, Lemma 1 establishes that the image of

$$
{ }_{l^{\prime}} E_{n+2-(m+1)}^{n+1,0} \rightarrow{ }_{v} E_{n+2-(m+1)}^{n+1,0}
$$

is finitely generated, completing the inductive step.

Setting $m=n$ in $(\dagger)$, the conclusion is that for each neighborhood $U$ of $y_{0}$, there is a neighborhood $V \subset U$ of $y_{0}$ such that the image of

$$
H^{n+1}\left(U ; \mathscr{H}^{0}\left[\left.f\right|_{f^{-1}\left(U^{\prime}\right)}\right]\right)={ }_{{ }^{\prime}} E_{2}^{n+1,0} \rightarrow{ }_{V} E_{2}^{n+1,0}=H^{n+1}\left(V ; \mathscr{H}^{0}\left[\left.f\right|_{f^{-1}(V)}\right]\right)
$$

is finitely generated. Since the sheaves that appear as coefficients in the cohomology are constant, with stalks a finitely generated free abelian group, it follows that the image of $H^{n+1}(U) \rightarrow H^{n+1}(V)$ is finitely generated as well. In combination with the fact that each of the individual elements of $H^{n+1}(U)$ is mapped to zero by the inclusion-induced homomorphism $H^{n+1}(U) \rightarrow H^{n+1}(W)$ for some neighborhood $W \subset U$ of $y_{0}$, it follows that $Y$ is $(n+1)$-clc at $y_{0}$.

The next result is an easy consequence of the preceding theorem and sets forth, perhaps in the most useful form, general criteria for detecting that cohomological local connectedness is preserved by a proper map.

Corollary 1. If $f: X \rightarrow Y$ is a proper map between locally compact metrizable spaces, the sheaves $\mathscr{H}^{k}[f]$ are locally constant for $k \leq n$, the groups $H^{k}\left(f^{-1}(y)\right)$ are finitely generated for $y \in Y$ and $k \leq n$, and $X$ is $\mathrm{clc}^{n+1}$, then $Y$ is clc $^{n+1}$. 


\section{SOME APPlications}

In order to make use of either Theorem 1 or its corollary, it is necessary to have machinery avaliable to detect that cohomology sheaves of mappings are locally constant. The three lemmas that follow provide the analyses needed for the applications. A more thorough presentation along these lines can be found in [DyW].

Lemma 2. If $f: X \rightarrow Y$ is a proper map between metrizable spaces and $V$ is an open subset such that:

(a) the inclusion-induced homomorphism $H^{k}\left(f^{-1}(V)\right) \rightarrow H^{k}\left(f^{-1}(y)\right)$ is an epimorphism for each $y \in V$, and

(b) the kernel of $H^{k}\left(f^{-1}(V)\right) \rightarrow H^{k}\left(f^{-1}(y)\right)$ is the same subgroup of $H^{k}\left(f^{-1}(V)\right)$ for each $y \in V$,

then the restriction of $\mathscr{H}^{k}[f]$ to $V$ is constant.

Proof. Specify a point $y_{0} \in V$. Conditions (a) and (b) imply that the quotient

$$
H^{k}\left(f^{-1}(V)\right) /\left(\operatorname{ker}\left\{H^{k}\left(f^{-1}(V)\right) \rightarrow H^{k}\left(f^{-1}\left(y_{0}\right)\right)\right\}\right)
$$

is "naturally" isomorphic to $H^{k}\left(f^{-1}(y)\right)$ for each $y \in V$. These isomorphisms combine to produce a map $p: V \times H^{k}\left(f^{-1}\left(y_{0}\right)\right) \rightarrow \mathscr{H}^{k}\left[\left.f\right|_{f^{-1}(V)}\right]$ that is determined by setting $p(y, \alpha)=$ image of $\tilde{\alpha}$ in $H^{k}\left(f^{-1}(y)\right)$, where $\tilde{\alpha} \in$ $H^{k}\left(\left(f^{-1}(V)\right)\right.$ is chosen so that $\alpha=$ image of $\tilde{\alpha} \in H^{k}\left(f^{-1}\left(y_{0}\right)\right)$. Evidently, $p$ is one-to-one and onto and it is a stalk-by-stalk isomorphism. The map $p$ is open since the image under $p$ of the basic open set $W \times\{\alpha\}$, for an open set $W \subset U$ and an element $\alpha \in H^{k}\left(f^{-1}\left(y_{0}\right)\right)$, is the basic open set consisting of the images of $\tilde{\alpha}$ in $H^{k}\left(f^{-1}(y)\right)$, for $y \in W$, where $\tilde{\alpha} \in H^{k}\left(f^{-1}(V)\right)$ is chosen so that the image of $\tilde{\alpha}$ in $H^{k}\left(f^{-1}\left(y_{0}\right)\right)$ is the element $\alpha$. It remains to show that the map $p$ is continuous. Specify a point $(y, \alpha) \in V \times$ $H^{k}\left(f^{-1}\left(y_{0}\right)\right)$. A typical basic open neighborhood of $p(y, \alpha)$, denoted $W^{*}$, consists of the images of $\tilde{\beta}$ in $H^{k}\left(f^{-1}(z)\right)$, for $z \in W$, where $W \subset V$ is an open subset, $y \in W$, and $\tilde{\beta} \in H^{k}\left(f^{-1}(W)\right)$. Let $\tilde{\alpha} \in H^{k}\left(f^{-1}(V)\right)$ be such that the image of $\tilde{\alpha}$ in $H^{k}\left(f^{-1}(y)\right)$ equals $\alpha$. The set $U=\{z \in$ $W$ : the images of $\tilde{\beta}$ and $\tilde{\alpha}$ in $H^{k}\left(f^{-1}(z)\right)$ are equal $\}$ is an open subset of $W$. Now, $U \times\{\alpha\}$ is a neighborhood of $(y, \alpha)$ and $p(U \times\{\alpha\}) \subset W^{*}$. Thus $p$ is continuous.

Recall that the collection of sections over a subset $A \subset Y$ of the cohomology sheaf $\mathscr{H}^{k}[f]$ associated to a map $f: X \rightarrow Y$ is denoted by $\Gamma_{k}(A)$.

Lemma 3. If $f: X \rightarrow Y$ is a proper map between metrizable spaces and the collection, denoted $\mathscr{P}$, consisting of those open sets $U \subset Y$ such that $\operatorname{ker}\left\{H^{k}\left(f^{-1}(U)\right) \rightarrow H^{k}\left(f^{-1}(y)\right)\right\}$ is the same subgroup of $H^{k}\left(f^{-1}(U)\right)$ for all $y \in U$ is a basis for $Y$, then the homomorphism $\phi_{y}: \Gamma_{k}(W) \rightarrow H^{k}\left(f^{-1}(y)\right)$ 
defined by $\phi_{y}(s)=s(y)$ is a monomorphism for each open connected set $W$ and each $y \in W$.

Proof. Suppose that $W$ is an open connected subset, $s \in \Gamma_{k}(W)$, and $s\left(y_{0}\right)=0$ for a point $y_{0} \in W$. It suffices to show that $W=\{z \in W: s(z)=0\}$. For each $y \in W$, specify an open set $V_{y} \subset W$, with $V_{y} \in \mathscr{S}$, such that there is an element $\alpha_{y} \in H^{k}\left(f^{-1}\left(V_{y}\right)\right)$ with the image of $\alpha_{y}$ in $H^{k}\left(f^{-1}(z)\right)$ equal to $s(z)$ for all $z \in V_{y}$. According to the hypothesis either $s(z)=0$ for each $z \in V_{y}$ or $s(z) \neq 0$ for each $z \in V_{y}$. Therefore the set $\{z \in W: s(z)=0\}$, which is necessarily open, is closed in $W$, as its complement is a union of $V_{y}$ 's. Thus, as $s\left(y_{0}\right)=0, W=\{z \in W: s(z)=0\}$.

Theorem 2. If $f: X \rightarrow Y$ is a proper surjection between locally compact metrizable spaces, the family of all open subsets $V \subset Y$ satisfying:

(a) $H^{k}\left(f^{-1}(V)\right) \rightarrow H^{k}\left(f^{-1}(y)\right)$ is an epimorphism for each $y \in V$, for $k=0$, and for each $k \leq n-1$, and

(b) $\operatorname{ker}\left\{H^{k}\left(f^{-1}(V)\right) \rightarrow H^{k}\left(f^{-1}(y)\right)\right\}$ is the same subgroup of $H^{k}\left(f^{-1}(V)\right)$ for each $y \in V$ and for $k \leq n$

is a basis for $Y$, and $X$ is $c l c^{n+1}$, then $Y$ is $c l c^{n}$ and $Y$ is $(n+1)$-clc at each point in the closure of

$$
F=\left\{y \in Y: H^{n}\left(f^{-1}(y)\right) \text { is finitely generated }\right\} .
$$

Proof. In view of Lemma 2 , the sheaves $\mathscr{H}^{k}[f]$ are locally constant for $k \leq$ $n-1$, and, as was discussed in $\S 2$, since $X$ is $\mathrm{clc}^{n}$, for an open set $V$ and point $y \in V$, the image of $H^{k}\left(f^{-1}(V)\right) \rightarrow H^{k}\left(f^{-1}(y)\right)$ is finitely generated for $k \leq n+1$. Thus, since those $V$ 's satisfying conditions (a) and (b) form a basis, $H^{k}\left(f^{-1}(y)\right)$ is finitely generated for each $y \in Y$ and $k \leq n-1$. It remains to show that condition $(*)$ of Theorem 1 is satisfied. Since the case $n=-1$ records that $Y$ is locally connected, Lemma 3 shows that condition $(*)$ holds at those points $y_{0} \in Y$, each neighborhood of which contains a point $y$ with $H^{k}\left(f^{-1}(y)\right)$ finitely generated, for $k \leq n$. While the latter holds at each point of $Y$ for $k \leq n-1$, it holds at the points of $F$ for $k=n$.

Theorem 3. If $f: X \rightarrow Y$ is a shape fibration between locally compact metrizable spaces, $X$ is clc ${ }^{n+1}$, and $H^{k}\left(f^{-1}(y)\right)$ is finitely generated for each $y \in Y$ and $k \leq n$, then $Y$ is $\mathrm{clc}^{n+1}$.

Proof. For an open set $U \subset Y$ and point $y_{0} \in U$, specify a connected open neighborhood $V$ of $y_{0}$ for which $H^{k}\left(f^{-1}(V)\right) \rightarrow H^{k}\left(f^{-1}\left(y_{0}\right)\right)$ is an epimorphism for $k \leq n$. For an arbitrary point $y \in V$, name an arc $A \subset V$ with endpoints $y_{0}$ and $y$. As recorded in [MR], the inclusions $f^{-1}(y) \hookrightarrow f^{-1}(A)$ and $f^{-1}\left(y_{0}\right) \hookrightarrow f^{-1}(A)$ are shape equivalences. Hence, $H^{k}\left(f^{-1}(A)\right) \rightarrow H^{k}\left(f^{-1}(y)\right)$ and $H^{k}\left(f^{-1}(A)\right) \rightarrow H^{k}\left(f^{-1}\left(y_{0}\right)\right)$ are isomorphisms for all $k$. It follows that $H^{k}\left(f^{-1}(V)\right) \rightarrow H^{k}\left(f^{-1}(y)\right)$ is an epimorphism with kernel equal to the kernel 
of $H^{k}\left(f^{-1}(V)\right) \rightarrow H^{k}\left(f^{-1}\left(y_{0}\right)\right)$ for $k \leq n$. Theorem 2 applies to detect that $Y$ is $\operatorname{clc}^{n+1}$.

\section{A CONVERSE TO THE PRINCIPAL RESULT}

Theorem 4 and Corollary 2 contain converses to Theorem 1 and Corollary 1 , respectively. In particular, the corollary records explicitly a fact alluded to earlier in the paper that, for proper maps defined on cohomologically locally connected spaces and having locally constant cohomology sheaves, the cohomological locally connectedness of the image forces the stalks to be finitely generated.

The proof of Theorem 4 uses the following extension of Lemma 0 that is a consequence of the universal coefficient theorem for Čech cohomology; for example, see [Sp, p. 335].

Lemma 4. If $G$ is a finitely generated group, $X$ is a locally compact space that is $\mathrm{clc}^{n}$, and $K \subset U$, where $K$ is compact and $U$ is open, then the image of $H^{k}(U ; G) \rightarrow H^{k}(K ; G)$ is finitely generated for $k \leq n-1$. Furthermore, if in addition $G$ is free, then the image of $H^{n}(U ; G) \rightarrow H^{n}(K ; G)$ is finitely generated as well.

Theorem 4. If $f: X \rightarrow Y$ is a proper map between locally compact metrizable spaces, $X$ is $c l c^{n+1}, Y$ is clc ${ }^{n}$, the cohomology sheaves $\mathscr{H}^{0}[f]$ as well as $\mathscr{H}^{k}[f]$, for $k \leq n-1$, are locally constant, and $Y$ is $(n+1)$-clc at a point $y$, then

(*) for each $k \leq n$ and each neighborhood $U$ of $y$, there is a neighborhood $V$ of $y$ such that the image of $\Gamma_{k}(U) \rightarrow \Gamma_{k}(V)$ is finitely generated (where $\Gamma_{k}(W)$ is the group of sections of $\mathscr{H}^{k}[f]$ over an open subset $\left.W \subset Y\right)$.

Proof. Assume that $Y$ is $(n+1)$-clc at $y_{0}$ and, replacing $Y$ by a neighborhood of $y_{0}$ if necessary, assume that each of the sheaves $\mathscr{H}^{0}[f]$ and $\mathscr{H}^{k}[f]$, for $k \leq n-1$, is constant. The proof is by induction on $0 \leq m \leq n$.

Suppose that $m=0$. Let $V \subset U$ a neighborhood of $y_{0}$. Adopting the notation used in the proof of Theorem 1,

$$
{ }_{{ }^{\prime}} E_{2}^{0,0}={ }_{{ }^{\prime}} E_{\infty}^{0,0} \hookrightarrow H^{0}\left(f^{-1}(U)\right) \text { and }{ }_{1}, E_{\infty}^{0,0} \hookrightarrow H^{0}\left(f^{-1}(V)\right) .
$$

Hence, if $V$ is chosen so that the image of $H^{0}\left(f^{-1}(U)\right) \rightarrow H^{0}\left(f^{-1}(V)\right)$ is finitely generated, then the image of ${ }_{U} E_{2}^{0,0}=H^{0}\left(U, \mathscr{H}^{0}\left[\left.f\right|_{U}\right]\right)=\Gamma_{0}(U)$ in ${ }_{1} E_{2}^{0,0}=H^{0}\left(V, \mathscr{H}^{0}\left[\left.f\right|_{{ }}\right]\right)=\Gamma_{0}(V)$ is finitely generated. Since $\mathscr{H}^{0}[f]$ is constant over $U$, it follows that $H^{0}\left(f^{-1}\left(y_{0}\right)\right)$ is finitely generated as well.

Inductively, assume that $1 \leq m \leq n-1$ and that, for each neighborhood $U$ of $y_{0}$, there is a neighborhood $V$ of $y_{0}$ contained in $U$ with the image of $\Gamma_{k}(U) \rightarrow \Gamma_{k}(V)$ finitely generated, for $k \leq m$. It follows that $H^{k}\left(y_{0}\right)$ is 
finitely generated as well. It follows from Lemma 4 that each neighborhood $W$ of $y_{0}$ contains a neighborhood $\alpha(W)$ of $y_{0}$ with $c l(\alpha(W)) \subset U$ such that the image of ${ }_{U} E_{2}^{p, q} \rightarrow{ }_{\alpha(U)} E_{2}^{p, q}$ is finitely generated, for either $p \leq m, q \leq m-1$ or $p=m+1, q=0$. It follows that

$$
\begin{gathered}
\text { for } r \geq 2 \text {, the image of }{ }_{U} E_{r}^{p, q} \rightarrow_{{ }_{\alpha}(U)} E_{r}^{p, q} \text { is finitely generated, } \\
\text { for either } p \leq m, q \leq m-1 \text { or } p=m+1, q=0 .
\end{gathered}
$$

It suffices to show that there is a neighborhood $V$ of $y_{0}$ such that the image of ${ }_{U} E_{r}^{0, m} \rightarrow{ }_{V} E_{r}^{0, m}$ is finitely generated for all $r \geq 2$, the case $r=2$ completing the inductive step. For any neighborhood $W,{ }_{W} E_{r}^{0, m}={ }_{W} E_{\infty}^{0, m} \subset$ $H^{m}\left(f^{-1}(W)\right)$, for $r \geq m+2$. Thus, for a neighborhood $V$ of $y_{0}$ with $\operatorname{cl}(V)$ a compact subset of $U$, since the image of $H^{m}\left(f^{-1}(U)\right) \rightarrow H^{m}\left(f^{-1}(V)\right)$ is finitely generated, the image of ${ }_{U} E_{r}^{0, m} \rightarrow{ }_{V} E_{r}^{0, m}$ is finitely generated for all $r \geq m+2$.

Proceeding by induction on $0 \leq p \leq m-1$, the inductive assumption is that for each neighborhood $\dot{U}$ of $y_{0}$, there is a neighborhood $\beta(U)$ such that the image of ${ }_{U} E_{m+2-p}^{0, m} \rightarrow{ }_{\beta(U)} E_{m+2-p}^{0, m}$ is finitely generated. Set

$$
\begin{gathered}
G={ }_{U} E_{m+2-(p+1)}^{0, m}, \quad H={ }_{\alpha(U)} E_{m+2-(p+1)}^{0, m}, \quad F=_{\beta\left(\alpha\left(U^{\prime}\right)\right)} E_{m+2-(p+1)}^{0, m}, \\
G_{1}=\operatorname{ker}\left({ }_{U} d_{m-p+1}^{0, m}\right), \quad H_{1}=\operatorname{ker}\left({ }_{\alpha(U)} d_{m-p+1)}^{0, m},\right.
\end{gathered}
$$

and let $f: G \rightarrow H$ and $g: H \rightarrow F$ be the inclusion-induced homomorphisms. Naturality assures that $f\left(G_{1}\right) \subset H_{1}$. Since $H_{1}=_{\alpha\left(U^{\prime}\right)} E_{m+2-p}^{0, m}$, the inductive assumption is that $g\left(H_{1}\right)$ is finitely generated. Since

$$
G / G_{1} \subset{ }_{U} E_{m+2-(p+1)}^{m-p+1, p} \text { and } H / H_{1} \subset{ }_{\alpha(U)} E_{m+2-(p+1)}^{m-p+1, p},
$$

condition (1) ensures that the image of $G / G_{1} \rightarrow H / H_{1}$ is finitely generated. Thus, Lemma 1 applies to show that

the image of ${ }_{U} E_{m+2-(p+1)}^{0, m} \rightarrow_{\beta(\alpha(U))} E_{m+2-(p+1)}^{0, m}$ is finitely generated, completing the inductive step.

Corollary 2. If $f: X \rightarrow Y$ is a proper surjective map between locally compact metrizable spaces, $X$ and $Y$ are $\mathrm{clc}^{n+1}$, and the cohomology sheaves $\mathscr{H}^{0}[f]$ as well as $\mathscr{H}^{k}[f]$, for $k \leq n$, are locally constant, then $H^{k}\left(f^{-1}(y)\right)$ is finitely generated for $k \leq n$ and $y \in Y$.

\section{REFERENCES}

[Ar] S. Armentrout, Homotopy properties of decomposition spaces, Trans. Amer. Math. Soc. 143 (1969), 499-507.

[AP] S. Armentrout and T. M. Price, Decompositions into compact sets with UV properties, Trans. Amer. Math. Soc. 141 (1969), 433-442. 
[Be] E. G. Begle, The Vietoris mapping theorem for bicompact spaces, Ann. of Math (2) 51 (1950), 534-543.

[Br] G. E. Bredon, Sheaf theory, McGraw-Hill, New York, 1967.

[CD] D. Coram and P. F. Duvall, Local n-connectivity and approximate lifting, Topology Appl. 13 (1982), 225-228.

[DaW] R. J. Daverman and J. J. Walsh, A cyclic decompositions of manifolds, Pacific J. Math. 109 (1983), 291-303.

[Dug] J. Dugundji, Modified Vietoris theorems for homotopy, Fund. Math. 66 (1970), 223-235.

[Dy 1 ] J. Dydak, Some properties of nearly 1-movable continua, Bull. Acad. Polon. Sci. Sér. Sci. Tech. 25 (1977), 685-689.

[Dy 2 ] _ On LC ${ }^{n}$-divisors, Topology Proc. 3 (1978), 319-333.

[DS] J. Dydak and J. Segal, Local n-connectivity of decomposition spaces, Topology Appl. 18 (1984), 43-58.

[DyW] J. Dydak and J. J. Walsh, Sheaves that are locally constant with applications to homology manifolds, Geometric Topology and Shape Theory, Springer-Verlag, New York, 1987, 65-87.

[Gr] A. Grothendieck, Sur quelques points d'algèbre homologique, Tĥoku Math. J. (2) 9 (1957), 119-221.

[Hu] W. Hurewicz, Homotopie, Homologie, und Lokaler Zusammenhang, Fund. Math. 25 (1935), 467-485.

[Koz] G. Kozlowski, Factorization of certain maps up to homotopy, Proc. Amer. Math. Soc. 21 (1969), 88-92.

[MR] S. Mardešić and T. B. Rushing, Shape fibrations, I, Gen. Topology Appl. 9 (1978), 193-215.

[Sm] S. Smale, A Vietoris theorem for homotopy, Proc. Amer. Math. Soc. 8 (1957), 604-610.

[Sp] E. H. Spanier, Algebraic topology, McGraw-Hill, New York, 1966.

[Sw] R. G. Swan, The theory of sheaves, Univ. Chicago Press, Chicago, 1964.

University of Tennessee, KNOXVille, Tennessee 37996

University of CALIFornia, Riverside, California 92521 